\title{
Minicore Myopathy with External Ophthalmoplegia
}

National Cancer Institute

\section{Source}

National Cancer Institute. Minicore Myopathy with External Ophthalmoplegia. NCI

Thesaurus. Code C150608.

An autosomal recessive condition caused by mutation(s) in the RYR1 gene, encoding ryanodine receptor 1. It may be characterized clinically by neonatal hypotonia, delayed motor development, and generalized muscle weakness, and amyotrophy. Pathologically, the absence of mitochondria and focal disorg anization of the sarcomere appear as "minicores" on AT Pase staining as a result of focal defects in oxidative activity. 